\title{
Ambio fit for the 2020s
}

\author{
Erik Andersson, Wiebren J. Boonstra, Maricela de la Torre Castro, \\ Alice C. Hughes, Ulrik Ilstedt, Arne Jernelöv, Bengt-Gunnar Jonsson, \\ Zahra Kalantari, Carina Keskitalo, Emma Kritzberg, Thomas Kätterer, \\ Jeffrey A. McNeely, Claudia Mohr, Tero Mustonen, Madelene Ostwald, \\ Victoria Reyes-Garcia, Graciela M. Rusch, Angelina Sanderson Bellamy, \\ Jesper Stage, Michael Tedengren, David N. Thomas, Angela Wulff, \\ Bo Söderström
}

Published online: 18 February 2022

Environmental challenges and the way we frame and research them are changing, as are the channels for successful transfer of scientific knowledge into decisionmaking. In a time when novelty is often the beacon leading funding, research and publication, revisiting the archives and going back to the roots of current discussions offer an opportunity to reflect on what got us to where we are today. Ambio's 50th anniversary in 2021 gave us a reason to look back at how Ambio's scope and coverage have changed over time and to start a discussion about how Ambio can remain relevant for science and policy in the future. Ambio is growing steadily, and we see an increasingly broad range of methodological approaches and use of different sources of evidence. This is encouraging; our ambition is to support multi- as well as inter- and transdisciplinary science on the interrelationships between the environment and society. At the same time, the field of sustainability science and applied social-environmental research is growing rapidly and with it comes many potential outlets for publishing work within Ambio's approximate niche. In this increasingly mixed and busy field, we work constantly to ensure that Ambio remains recognized as a leading forum for high quality science, based on a broad foundation of knowledge and at the interface of society and the environment.

Where are we in this work? In 2021 we discussed with our advisory board Ambio's aims and scope, article formats, the editorial and peer review processes and more broadly where the field of sustainability science and the study of social-environmental interactions is moving. In this editorial we will outline some of the changes that stem from these discussions. Some of these we have already implemented and others we will try out during 2022.

First, we have updated the Ambio author guidelines to explicitly state that "our articles present research to help understand contemporary sustainability changes and how proposed solutions may be implemented", a change also reflected in the new subtitle "A Journal of Environment and Society". Furthermore, we emphasize that Ambio (1) publishes research on the interrelationships between the environment and society and (2) encourages multi-, interand trans-disciplinary research with explicit management and policy recommendations. To accommodate different writing traditions and disciplinary standards we now offer an opportunity to include an explicit 'Theoretical framework section' for clarifying theoretical assumptions that might not always be evident to our broad readership. We also emphasize that one of the core qualities we look for in a submission is a discussion about the discipline-specific and cross-disciplinary novelty of the study in relation to the existing body of literature that it engages with.

Second, the increasing recognition of human influence on all biophysical processes-together with our aim to increasingly promote interdisciplinarity - is reflected in the composition of our editorial board. We now have more editors with backgrounds in the social sciences and humanities and we are continuously reviewing our editorial work to ensure Ambio's quality and scientific rigour. Here, we have identified a couple of priority areas, including continued work to ensure methodological robustness of all publications, from targeted disciplinary basic research to solution-oriented applied transdisciplinary work. The strategy for making improvements within the priority areas includes greater communication between editors and advisory board members, tapping into the broad and deep collective expertise, and internal discussions on 'scientific quality' and standards for submissions coming from different disciplines and traditions.

Third, another priority for the editorial team is to make sure policy recommendations and suggested practical solutions are robustly grounded in evidence. Insights from, 
among other fields, basic qualitative social science, actionoriented research and knowledge co-production, along with recognition of non-scientific knowledge systems and expertise, have led to an improved interdisciplinary and pluralistic understanding of how different stakeholders can and need to be involved in a social-environmental problem solving. An increasing number of journals and other dissemination venues make claims to supporting this development and being open to consider evidence from different knowledge systems, but the support needs to be backed by carefully developed standards, documentation protocols and procedures understood as legitimate by academia as well as the rest of the society. This is particularly critical in a time of political populism, anti-science movements, and active misinformation campaigns. We will continue to investigate ways to ensure (e.g. by, possibly, inviting nonacademic reviewers to look at suggested 'solutions') that all contributions to Ambio are critically and fairly examined, from the evidence base and analyses to the feasibility and grounding of the conclusions and recommendations.

Fourth, we believe there is a need for a new forum for cross-examining and contrasting important ideas, concepts, and results of research embedded in complex social-environmental contexts. The backbone of the $50^{\text {th }}$ anniversary volume was formed by the collection of anniversary thematic articles featuring environmental problems such as decomposition of the ozone layer and biodiversity conservation, as well as broad phenomena like urbanization and the Anthropocene. Novel to Ambio, the anniversary collections combined author reflections with peer perspectives in a critical cross-examination, providing an opportunity to both look back at how our understanding has grown, and to take stock of where we are today and look forward. We will continue to explore this space for multiple interpretations through a new initiative called thematic features, actively curated by Ambio editors and advisory board members. We have decided not to introduce any new article types, instead we will continue to make use of our Perspective format. Perspectives are full-length and peer reviewed contributions that provide a forum for authors to propose alternative ways of synthesizing and analysing already published evidence and share thoughts on topical environmental issues, ideas, or models based on their understanding of the field. About $20 \%$ of all published articles in 2019 were Perspectives and the relatively high number of citations (on average 14.25 compared to 9.18 for research articles, see Fig. 1) give clear evidence that this article category is in demand.

The new thematic features will be centred around 2-3 Perspective papers (for the first thematic feature by invitation only, later potentially 1-2 commissioned papers plus 1 contribution from an open invitation) offering different approaches and ways of looking at an issue. These will be

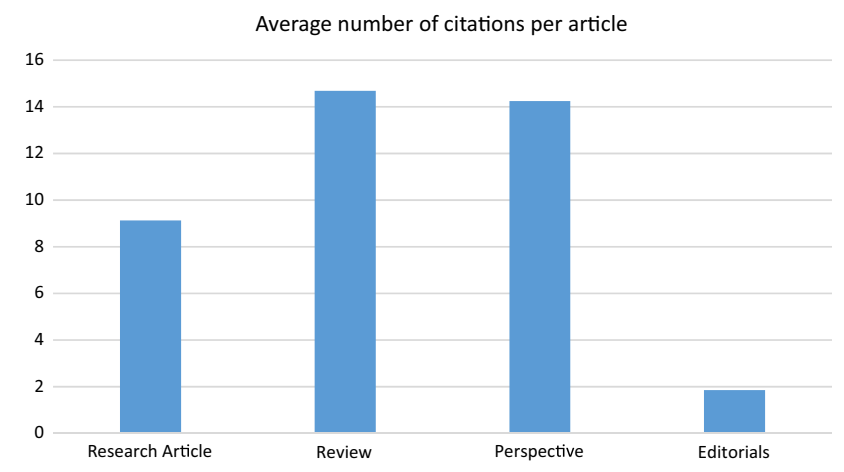

Fig. 1 Average number of citations per article category based on volumes 48, 49 and first three issues of volume 50 (based on 218 Research articles, 48 Reviews, 49 Perspectives and 14 Editorials). Data retrieved from Clarivate Analytics on December 20, 2021

accompanied by a minimum of two Comments reflecting on and adding to the Perspectives. Pre-proofs/proofs of the Perspectives will be made available to editors and advisory board members, who will be asked to add a first round of Comments reflecting on the points made in the Perspectives. These Comments will be included in the thematic feature, and, as with all publications, additional Comments can be submitted at any time after the thematic feature has been published for later publication and a continued discussion. The final thematic feature will be introduced by a longer Editorial.

The 50th anniversary has come and gone. But as this editorial has made clear-this was not 'it', the work continues. We have gained new inspiration through the anniversary celebrations to continue publishing high-quality, relevant and multi-, inter-, and transdisciplinary science for sustainability in the years to come. The outlined changes in the Ambio editorial work we hope will help us achieve our goals of contributing with robust, pluralist, and actionable knowledge to policy and decision-making for taking better care of our planet.

Publisher's Note Springer Nature remains neutral with regard to jurisdictional claims in published maps and institutional affiliations.

Erik Andersson ( $\square)$

Address: Stockholm Resilience Centre, Stockholm University, 106 91, Stockholm, Sweden.

Address: Unit for Environmental Sciences and Management, NorthWest University, Potchefstroom 2520, South Africa.

e-mail: erik.andersson@su.se

\section{Wiebren J. Boonstra}

Address: Natural Resources and Sustainable Development, Department of Earth Sciences, Uppsala University, 75236 Uppsala, Sweden e-mail: wijnand.boonstra@geo.uu.se 
Maricela de la Torre Castro

Address: Department of Physical Geography, Stockholm University, 10691 Stockholm, Sweden.

e-mail: maricela@natgeo.su.se

\section{Alice C. Hughes}

Address: School of Biological Sciences, The University of Hong Kong, Hong Kong, China.

e-mail: ach_conservation2@hotmail.com

\section{Ulrik Ilstedt}

Address: Department of Forest Ecology and Management, Swedish University of Agricultural Sciences, 90183 Umeå, Sweden.

e-mail: ulrik.ilstedt@slu.se

\section{Arne Jernelöv}

Address: Stockholm, Sweden.

e-mail: arne.jernelov@gmail.com

\section{Bengt-Gunnar Jonsson}

Address: Department of Natural Sciences, Mid Sweden University, 85170 Sundsvall, Sweden.

Address: Department of Fish, Wildlife and Environmental Science, Swedish University of Agricultural Sciences, 90183 Umeå, Sweden. e-mail: bengt-gunnar.jonsson@miun.se

\section{Zahra Kalantari}

Address: Department of Physical Geography and Bolin Centre for Climate Research, Stockholm University, 10691 Stockholm, Sweden Address: Department of Sustainable Development, Environmental Science and Engineering, KTH Royal Institute of Technology, 10044 Stockholm, Sweden.

e-mail: zahra.kalantari@natgeo.su.se

\section{Carina Keskitalo}

Address: Department of Geography, Umeå University, 90187 Umeå, Sweden.

e-mail: carina.keskitalo@umu.se

\section{Emma Kritzberg}

Address: Department of Biology, Lund University, 22362 Lund, Sweden.

e-mail: emma.kritzberg@biol.lu.se

\section{Thomas Kätterer}

Address: Department of Ecology, Swedish University of Agricultural Sciences, 75007 Uppsala, Sweden.

e-mail: thomas.katterer@slu.se

\section{Jeffrey A. McNeely}

Address: Society for Conservation Biology Asia Section, Petchburi, Thailand.

e-mail: jeffmcneely2@gmail.com

\section{Claudia Mohr}

Address: Department of Environmental Science, Stockholm University, 106 91, Stockholm, Sweden.

e-mail: claudia.mohr@aces.su.se
Tero Mustonen

Address: Snowchange Cooperative, Lehtoi, Finland.

e-mail: tero@lumi.fi

\section{Madelene Ostwald}

Address: Department of Technology, Management and Economics, Chalmers University of Technology, 41296 Gothenburg, Sweden.

Address: Gothenburg Centre for Sustainable Development, 40530 Gothenburg, Sweden.

e-mail: madelene.ostwald@chalmers.se

\section{Victoria Reyes-Garcia}

Address: Institució Catalana de Recerca I Estudis Avançats (ICREA), 08010 Barcelona, Spain.

Address: Institut de Ciència i Tecnologia Ambientals, Universitat Autònoma de Barcelona (ICTA-UAB), 08193 Barcelona, Spain.

e-mail: victoria.reyes@uab.cat

\section{Graciela M. Rusch}

Address: Norwegian Institute for Nature Research, 7485 Trondheim, Norway.

e-mail: graciela.rusch@nina.no

\section{Angelina Sanderson Bellamy}

Address: Department of Applied Sciences, University of the West of England at Bristol, Bristol, UK.

e-mail: angelina.sandersonbellamy@uwe.ac.uk

\section{Jesper Stage}

Address: Department of Social Sciences, Technology and Arts, Luleå University of Technology, 97187 Luleå, Sweden.

e-mail: jesper.stage@1tu.se

\section{Michael Tedengren}

Address: Department of Ecology, Environment and Plant Sciences, Stockholm University, 106 91, Stockholm, Sweden.

e-mail: michael.tedengren@su.se

\section{David N. Thomas}

Address: University of Helsinki, 00014 Helsinki, Finland.

e-mail: david.thomas@helsinki.fi

\section{Angela Wulff}

Address: Biological and Environmental Sciences, University of Gothenburg, 40530 Gothenburg, Sweden.

e-mail: angela.wulff@bioenv.gu.se

\section{Bo Söderström}

Address: The Royal Swedish Academy of Sciences, 10405 Stockholm, Sweden.

e-mail: bo.soderstrom@kva.se 